\title{
Innovaciones en las políticas nacionales de Educación de Jóvenes y Adultos (EJA): el Programa FINES en Argentina y el PROEJA en Brasil
}

\author{
Mónica de la Fare* \\ Laura Rovelli** \\ Daniela Atairo*** $^{* *}$
}

\begin{abstract}
Resumen
Este artículo focaliza en la identificación de nuevas tendencias en las políticas de EJA, en Argentina y en Brasil. En un primer momento contextualizamos brevemente aspectos históricos de los sistemas educativos de cada país, centrándonos en la EJA y enfatizando contrastes entre los dos países. Posteriormente, avanzamos en el análisis de dos programas implementados en los años 2000, que buscan producir innovaciones en la Educación de Jóvenes y Adultos: El Plan FINES en Argentina y el PROEJA en Brasil. Para la realización de este trabajo recurrimos principalmente al análisis de documentos oficiales de política educativa de cada país y a las contribuciones de estudios especializados en el tema.
\end{abstract}

Palabras-clave: Educación de Jóvenes y Adultos; Argentina; Brasil; FINES; PROEJA.

\section{Innovations in national policies of Education for Youth and Adults (EJA): FINES Program in Argentina and PROEJA in Brazil}

\begin{abstract}
This article focuses on identifying new trends in EJA policies in Argentina and Brazil. At first briefly we contextualize historical aspects of education systems in each country, focusing on the EJA and emphasizing contrasts between the two countries. Then we move into the analysis of two implemented in late 2000 programs, which seek to produce innovations in Youth and Adult Education: The Plan FINES in Argentina and the PROEJA in Brazil. To carry out this work mainly resorted to the analysis of official documents of educational policy of each country and and the contributions of studies on the subject.

Keywords: Youth and Adult Education; Argentina; Brazil; FINES; PROEJA.

\section{Inovações em políticas nacionais de Educação de Jovens e Adultos (EJA): O Plano FINES na Argentina y o PROEJA no Brasil}

\section{Resumo}

Este artigo focaliza na identificação de novas tendências nas políticas de EJA, na Argentina e no Brasil. Primeiramente, contextualizamos brevemente aspectos históricos dos sistemas educativos de cada país, enfatizados na EJA e nos contrastes entre os dois países. Posteriormente, o artigo apresenta uma análise dos programas implementados nos últimos anos da década do 2000. O Plano FINES na Argentina e o PROEJA no Brasil. Para a realização deste trabalho utilizamos principalmente a análise de documentos oficiais da política educativa de cada país e estudos especializados sobre o tema.

Palavras-chave: Educação de Jovens e Adultos; Argentina; Brasil; FINES; PROEJA.

La tarea realizada en el trabajo que presentamos a continuación exige algunos esclarecimientos en relación a la opción por problematizar aspectos que consideramos innovadores en las políticas en Educación de Jóvenes y Adultos (EJA) en dos países de la región sudamericana: Argentina y Brasil ${ }^{1}$. En las últimas décadas ambos países ampliaron su cobertura escolar e implementaron políticas de democratización del acceso y la permanencia a los

\footnotetext{
* Endereço Eletrônico: monica.fare @ pucrs.br

*** Endereço Eletrônico: laurarovelli@gmail.com

**** Eletrônico Eletrônico: atairodaniela@gmail.com
}

sistemas educativos de las clases sociales menos favorecidas de la sociedad. En ambos contextos se avanzó, a través de normativas nacionales, en la extensión del número de años de escolaridad obligatoria. En Brasil, la Emenda Constitucional ${ }^{\circ}$ 59, del 11 de noviembre de 2009 estableció la educación básica obligatoria y gratuita de los cuatro (4) a los diecisiete años (17) así como la oferta gratuita de dicha escolaridad para quienes "no tuvieron acceso en la edad propia" (BRASIL, 2009). 
Esa obligatoriedad en Argentina fue establecida por la Ley $\mathrm{n}^{\mathrm{o}} 26.206$ de 2006, denominada Ley de Educación Nacional (LEN): "La obligatoriedad escolar en todo el país se extiende desde la edad de cinco (5) años hasta la finalización de la Educación Secundaria (ARGENTINA, 2006). Posteriormente, esa normativa fue modificada por la Ley $\mathrm{n}^{\circ} 27.045$, del 3 de diciembre de 2014, que pauto el inicio de escolaridad obligatoria a partir de los cuatro (4) años (ARGENTINA, 2014). La LEN también reconoció a la EJA como una de las ocho modalidades educativas del sistema educativo nacional, cuestión que ya había sido inicialmente establecida en Brasil por la Ley de Directrices y Bases de la Educación Nacional de 1996 (LDB), posteriormente reforzada por las Directrices Curriculares para la Educación de Jóvenes y Adultos (Parecer CNE/CEB n ${ }^{\circ}$ 11/2000).

En este marco, ambos países, a partir de configuraciones históricas distintas de los sistemas educativos produjeron, a partir de la segunda mitad de los años 2000, modificaciones en las ofertas educativas destinadas a la Educación escolar de Jóvenes y Adultos. Desde ese punto de vista entendemos al Plan de Finalización Plan de Finalización de estudios Primarios y Secundarios (PLAN FINES) para jóvenes y adultos, de Argentina y al Programa Nacional de Integración de la Educación Profesional con la Educación Básica en la modalidad EJA (PROEJA), en Brasil. Se trata de acciones implementadas como posibilidad de superación de una tradición asociada a la educación compensatoria y remedial, presente desde los inicios de la institucionalización de servicios educativos estatales para la EJA en los dos países. Más que comparar las orientaciones de dichos programas, buscamos identificar exploratoriamente algunos contrastes que permitan reconocer sus especificidades. Para eso, los abordamos como casos de innovación de las políticas de la modalidad educativa EJA en la región sudamericana y recurrimos principalmente al análisis de documentos oficiales $y$ al estudio de las contribuciones de las producciones especializadas sobre el tema.

Aclaramos que este análisis se extiende hasta el año 2015, los nuevos rumbos políticos experimentados en la Argentina y en Brasil marcan una ruptura, en términos de un retroceso, en relación al conjunto de políticas públicas, incluidas las educativas. Por la inmediatez de esta coyuntura resolvimos no avanzar en los análisis del momento actual y restringir nuestro alcance temporal.

A continuación, presentamos este texto organizado en dos grandes apartados, en el primero contextualizamos brevemente aspectos históricos de la institucionalización de la EJA en ambos países. Esclarecemos que no realizamos un trabajo de reconstrucción histórica de la EJA en la Argentina o en Brasil, varias producciones que anteceden a este trabajo desarrollan y profundizan ese abordaje. Nos limitamos a considerar particularidades de esa modalidad educativa en los sistemas educativos, para comprender las orientaciones priorizadas por las novedades introducidas en las ofertas de la EJA. En el segundo apartado, presentamos y analizamos algunas de las principales características del FINES y del PROEJA.

\section{Contrastes entre la EJA en la Argentina y en Brasil: configuraciones históricas de los sistemas educativos nacionales}

A comienzos del siglo XX, en Argentina, el Estado Nacional se constituyó en el principal agente educativo al garantizar el derecho a la educación primaria a lo largo del territorio nacional y diseñar e implementar las directrices en torno a la formación docente, política curricular y gobierno del sistema. Aunque el sistema educativo argentino asumió en sus orígenes -siguiendo el modelo europeo- una estructura por niveles y funciones diferentes de acuerdo a los distintos sectores sociales. Logró así expandir y consolidar tempranamente una escuela primaria única y común a toda la población con una amplia cobertura. Esa dinámica expansiva del nivel primario se tradujo en una demanda de continuidad de estudios en el nivel secundario y superior por parte de los sectores sociales medios en ascenso. A pesar de que el circuito de los colegios nacionales y de la universidad estaba reservado para los sectores medio altos y altos, la dinámica de movilidad ascendente permitió generar la expectativa de acceso a la clase media (TIRAMONTI; SUÁSNABAR, 2000). Según el censo de 1914, el $48 \%$ de los niños entre 6 y 14 años de edad se encontraban matriculados en la escuela primaria, cifra que hacia 1947 se incrementó al $75 \%$. Mientras que la tasa de escolarización del grupo de edad de entre 13 y 18 años, alcanzó el $3 \%$ en 1914 , el $10 \%$ en 1943 y en 1960 ya se había más que duplicado (23\%) (FERNÁNDEZ; LEMOS; WIÑAR, 1997). En este contexto, EJA se configuró como un sistema compensatorio para quienes no han logrado completar sus estudios y se equipara 
con la educación primaria que tendía a universalizarse (HISSE, 2009).

A diferencia de la Argentina, la amplia expansión de la Educación Primaria en Brasil (nivel educativo denominado en ese país como Enseñanza Fundamental) se concretizó mucho más tardíamente, a fines del siglo XX, cuestión vinculada al papel del Estado en relación a las políticas educativas. Recién entre 1975 y 2002 la matrícula total de ese nivel educativo creció 71,5\%, aumentando de 19,5 millones para 33,5 millones (OLIVEIRA, 2007). Un importante incremento de matrículas, especialmente las de los primeros años del nivel primario, se produjo entre 1970 y 1980 . En la década de 1990 también se verificó un aumento de matrículas, particularmente las de los años finales de ese nivel educativo y del nivel medio.

El censo nacional de 1920 indicó que $72 \%$ de la población con más de cinco años se encontraba en condición de analfabetismo y cuatro décadas después, ese índice aún era del 46,7\% (HADDAD; DI PIERRO, 2000). La evolución de ese indicador muestra una marcada mejora de esa situación pasadas otras cuatro décadas. El Censo Nacional del 2000 mostró, para la población de 15 años y más, una tasa de analfabetismo del $12,8 \%$ en ese año y en el de 2010 de un 9\% (OLIVEIRA, 2007). Los datos disponibles del IBGE/PNAD mostraron que $8,3 \%$ de la población brasileña en 2014 se declaró analfabeta para los censistas, representando aproximadamente 13 millones de personas (BRASIL, 2016).

Contrastando con Argentina, que incluyó la creación de escuelas para adultos en la primera ley nacional de instrucción pública (Ley N 1420 de Educación Común de 1884), los primeros servicios educativos estatales en Brasil fueron institucionalizados en la década de 1940, a través de la creación del fondo Nacional de Enseñanza Fundamental en 1942, reglamentado en 1945 y de la creación del Servicio de Educación de Adultos (SEA) del Departamento de Educación del Ministerio de Salud en 1942. Sin embargo, la realización de campañas masivas de alfabetización se inició en 1947, antes que en la Argentina, y el organismo responsable por esas acciones fue el SEA, siendo reconocida la influencia de los lineamientos de la UNESCO en estas iniciativas (DE LA FARE, 2011). Beisegel (1997) y Haddad (1999) apuntan que esa campaña, realizada a partir de la articulación de acciones entre la Unión, los territorios y los Estados permitió crear en casi todas las unidades de la federación servicios educativos para adultos. Posteriormente, en la década del 50, se organizaron otras campañas de alfabetización, como la Campaña Nacional de Educación Rural en 1952 y la de Erradicación del analfabetismo en 1958, que tuvieron una corta duración y un alcance limitado (HADDAD; DI PIERRO, 2000).

Se identifica la influencia de la UNESCO como orientadora de esas acciones masivas de alfabetización en varios países de la región. Si bien ese organismo internacional no priorizó acciones de financiamiento, influenció las políticas educativas de los países a través de la realización de eventos de amplia escala (FERREIRA; FONSECA, 2011). En ese sentido, un claro ejemplo de su actuación son las Conferencias Internacionales de Educación de Jóvenes y Adultos (CONFINTEA), organizadas por la UNESCO desde 1948, con una periodicidad aproximada de $10 \quad$ o 12 años. La última CONFINTEA fue realizada en Belén do Pará, Brasil, en 2009.

A las acciones educativas que venía implementando el Estado Nacional en Argentina en torno al proceso de expansión del sistema educativo, desde inicios del siglo $\mathrm{XX}$ se sumaron con gran dinamismo los estados provinciales y el sector privado. Con ello, se produjo una progresiva diferenciación del nivel secundario durante las décadas del 1950 y del 1960: a los tradicionales bachilleres, las escuelas normales y las comerciales se agregó el desarrollo de orientaciones técnicas con el propósito de integrar a los sectores populares en nuevos circuitos educativos, orientados a la educación para el trabajo (SUASNÁBAR; ROVELLI, 2010).

Para la EJA, la década de 1960 en Argentina representó el momento de implementación de la primera campaña estatal masiva de alfabetización, llamada Programa Intensivo de Alfabetización y Educación de Adultos (PIAyEA), a partir de la creación centros educativos específicos y con un sistema de trabajo diferente al de las escuelas convencionales (DE LA FARE, 2010). Como ya fue señalado, a diferencia de Brasil, este país adopta esa política más de una década después, probablemente por las aproximaciones del gobierno de Arturo Illia (1963-1966) a la UNESCO y por la influencia de Luz Vieyra Méndez, pedagoga y presidente del Consejo Nacional de Educación en esos años, quien había trabajado anteriormente como experta de ese organismo internacional (DE LA FARE, 2013).

Con el impulso del PIAyEA se crea la Dirección Nacional de Alfabetización y Edificación 
Escolar, que en 1966 pasó a denominarse Dirección Nacional de la Campaña de Alfabetización. Posteriormente, ya en un régimen dictatorial, ese organismo se transforma y en 1968 se funda la Dirección Nacional de Educación de Adultos (DINEA), de la cual pasaron a depender las escuelas para adultos. Unos años después, en 1970, a partir de ese espacio se institucionalizan varios centros educativos para la atención de necesidades específicas, entre ellos los Centros Educativos de Nivel Secundario. Esa Dirección Nacional tiene posteriormente un papel protagónico durante los pocos años de democracia argentina de la década de 1970. En un corto periodo se diseñó a partir de ese organismo estatal la denominada Campaña de Reactivación Educativa de Adultos para la Reconstrucción (CREAR), en parte inspirada por las ideas de Paulo Freire, quien había publicado en 1970, durante su exilio en Chile su reconocido libro, Pedagogía del Oprimido, rápidamente divulgado en español en varios países. La CREAR, interrumpida por la dictadura militar, tuvo una breve implementación y no llegó a efectivizar la mayoría de sus propuestas. Situación similar a la experimentada en Brasil por el Plan Nacional de Alfabetización, coordinado por el mismo Paulo Freire a fines de 1963 y abortado por el golpe de estado de 1964.

En Brasil, a fines de la década de 1950, existieron indicios de un importante movimiento de renovación pedagógica en la EJA, que se acentuaron en los primeros años de la década de 1960, momento de una fuerte efervescencia política (HADDAD; DI PIERRO, 2000). Esa década representó una etapa marcada por contradicciones, avances y marcados retrocesos. Hasta el golpe de estado de 1964 ganaron espacio un conjunto de iniciativas vinculadas a iniciativas de la sociedad civil, encabezadas por diversos espacios y movimientos de educación y cultura popular. En ese contexto los trabajos en Educación de Adultos con los sectores populares constituyeron formas de sustentación política de las propuestas de esos grupos (HADDAD; DI PIERRO, 2000).

El golpe militar de 1964 exterminó estas iniciativas e impuso una política de EJA que combinó la creación del Movimiento Brasilero de Alfabetización (MOBRAL), a partir de la Ley $\mathrm{n}^{\circ}$ 5.379, del 15 de diciembre de 1967 con la institucionalización de la Enseñanza Supletoria, a partir de la Lei $\mathrm{n}^{\circ}$ 5.692, del 11 de agosto de 1971, denominada Ley de Diretrices y Bases de la Educación Nacional. Ambas propuestas, de carácter tecnicista, orientaron la EJA hacia la formación para el mercado de trabajo y a la mejora de los indicadores de analfabetismo de jóvenes y adultos.

También los sombríos años de los regímenes cívico-militares en Argentina se caracterizaron por políticas de subsidiariedad estatal y su consecuente transferencia de gestión y financiamiento de las escuelas, especialmente de nivel primario a las jurisdicciones. El efecto que tuvieron dichas políticas fue el de configurar un sistema educativo segmentado y desarticulado que tendía a reproducir las desigualdades sociales (BRASLAVSKY, 1985). Las escuelas para adultos experimentaron un fuerte deterioro a partir de la disminución de la oferta educativa, de la matrícula y la reducción del número de docentes. Durante el período de transición democrática, si bien se logró consensuar la necesidad de reforma del sistema educativo, se dilataron las iniciativas de cambio en un escenario de graves crisis económicas hacia finales de la década.

En 1988, después de casi dos décadas de gobiernos militares, fue promulgada en Brasil una nueva Constitución Federal que reconoció el derecho a la educación como un derecho público subjetivo, independiente de la edad. El artículo $37^{\circ}$ declara: "La educación de jóvenes y adultos será destinada a aquellos que no tuvieron acceso $u$ oportunidad de estudios en la enseñanza fundamental y media en la edad propia" (BRASIL, 1988). Posteriormente, la LDB de 1996 la reconoce como modalidad educativa y en el año 2000 las Directrices Curriculares de la Educación de Jóvenes y Adultos (Parecer CNE/CEB 11/2000) reafirmaron esa condición enfatizando la necesidad de un abordaje acorde a sus especificidades. Esa normativa también reconoce para la EJA el carácter de deuda social no reparada para quienes no tuvieron acceso ni dominio de la escritura y la lectura como bienes sociales en la escuela o fuera de ella (BRASIL, 2000, p.5).

En los avances de la década de 1990, el gobierno de Fernando Henrique Cardoso impulsa una importante reforma del gobierno brasileño incluyendo una política de privatizaciones de empresas públicas, e iniciativas de regulación de la Administración Federal a través de un modelo de descentralización, en el marco de la estabilidad lograda con el Plan Real, con importantes repercusiones en el sistema educativo y con medidas que contrariaron los derechos y garantías conquistados en la Constitución Federal de 1988 y establecieron nuevas formas de financiamiento, 


\section{en Argentina y el PROEJA en Brasil}

gestión y evaluación de la educación, siguiendo las tendencias reconocidas en el ámbito internacional (OLIVEIRA, 2009). Un ejemplo de esto fue la institucionalización del Fondo de Desenvolvimiento de la Enseñanza Fundamental y la Valorización del Magisterio (FUNDEF), que indujo a la municipalización de la enseñanza fundamental y dejó parcialmente descubierto el financiamiento de tres segmentos de la educación básica: la Educación Infantil, el Nivel Medio y la Educación de Jóvenes y Adultos (HADDAD; DI PIERRO, 2000). La EJA ocupó un lugar secundario en la reforma de la educación brasileña impulsada en la segunda mitad de la década de 1990, dadas las estrategias de focalización de recursos en los servicios educativos direccionados a la infancia y a la adolescencia. Como apunta Di Pierro (2005), rescatando los avances experimentados en materia normativa, el problema no estaba en las leyes y sí en las políticas educativas, más allá de la existencia de un marco jurídico adecuado.

También en esos años y como forma de organización para participar de la V CONFINTEA, realizada en 1997 en Hamburgo, Alemania, se inició en Río de Janeiro una experiencia de organización de un Foro de Educación de Jóvenes y Adultos. Posteriormente esos Foros se expandieron por todos los Estados de Brasil y constituyeron espacios de discusión de políticas en EJA de los que participan representantes de diferentes sectores (gestores vinculados a la EJA en municipios y Estados, movimientos sociales, universidades, organizaciones no gubernamentales, etc.). Actualmente, cada Estado cuenta con un Foro de EJA y son espacios a partir de los cuales se organizan encuentros y seminarios nacionales de EJA. La EJA en Argentina no cuenta con un espacio semejante.

La década de 1990 fue marcada en la región por el auge del ideario neoliberal y la imposición de un modelo de Estado mínimo, reducido principalmente en relación a las políticas sociales. En materia de políticas educativas, los organismos internacionales, principalmente el Banco Mundial y el Banco Interamericano de Desarrollo, tuvieron un papel protagónico en relación a la reorientación y financiamiento de políticas educativas en varios países de América Latina. La gestión educativa en estos años fue predominantemente orientada por un modelo gerencial sustentado en lógicas de mercado y criterios de eficiencia. Aunque la agenda mundializada de la educación paute las políticas de los países, la forma en que las políticas se concretaron presenta diferencias vinculadas a la historia y a las fuerzas políticas en disputa en cada uno de ellos. En este contexto, la Argentina se convirtió en uno de los principales laboratorios de las políticas neoliberales en la región sudamericana, con las consecuencias evidenciadas en el estallido social, producido en el año 2001.

En este escenario la nueva reforma del sistema educativo en la Argentina llegó de la mano de un nuevo rol que asumió el Estado Nacional en la conducción del sistema educativo y de nuevos paradigmas de políticas de orientación neoliberal. En los primeros años de la década de 1990 se sancionó un paquete legislativo que reconfiguró el sistema educativo: la Ley de Transferencia de Servicios Educativos del nivel medio y superior no universitario $\mathrm{n}^{\circ} 24.049$ de 1992 , la Ley Federal de Educación, $\mathrm{n}^{\circ}$ 24.195, de 1993 y la Ley de Educación Superior $n^{\circ} 24.521$, de 1995. La sanción de la normativa estuvo acompañada por la implementación de un conjunto de políticas que fortalecieron las tendencias de cambio: de evaluación, que permitieron un control "a distancia" de los actores educativos; focalizadas en las escuelas con población socioeconómica desfavorecida; curriculares y de formación docente. Durante este período se extendió la obligatoriedad de la educación a diez años y se modificó la estructura tradicional del sistema sin perder su carácter selectivo. Como resultado, se implementó una Escuela General Básica (EGB) de nueve años obligatorios y un ciclo llamado "polimodal", de especialización no obligatorio. La tasa de escolarización bruta del nivel secundario mostró un incremento sustantivo: en 1980 alcanzaba el 63\%, en 1991 el $72 \%$ y en el 2001 el $85 \%$ (ARGENTINA, 2003).

En este escenario, la Educación de Jóvenes y Adultos (EJA) tuvo un reconocimiento parcial en el sistema educativo formal al considerarla dentro de los "regímenes especiales", semejante a la Educación Especial y a la Educación Artística. Al escaso reconocimiento de la particularidad y de las problemáticas específicas de la EJA se sumó el ciclo final del proceso de transferencia de los servicios educativos a las jurisdicciones provinciales, motorizado previamente por las políticas educativas de la última dictadura cívicomilitar de los años 70. Con todo, la EJA contó con recursos escasos y dispares para su desarrollo de acuerdo a las distintas jurisdicciones.

Haciendo foco en las políticas educativas de la última década, corresponde destacar que, en el 
año 2006, en la Argentina, la sanción de la Ley de Educación Nacional, n²6.206 (LEN), derogó la sancionada en 1993 y pretendió revertir los efectos negativos que había generado la implementación de una reforma de corte neoliberal, a partir de invertir algunos de sus sentidos ordenadores. Entre ellos, destaca la concepción de la educación como un bien público y un derecho social y la centralidad que cobra el Estado Nacional en garantizar ese derecho. Es más, la nueva legislación estableció como finalidad garantizar a la alfabetización y el cumplimiento de la obligatoriedad escolar hasta la finalización del nivel secundario (trece años en total). Esta ley fue la más importante dentro de un paquete legislativo que recién hacia fines de la primera década de 2000 estuvo acompañado de acciones e instrumentos de política que garanticen los cambios propuestos (FELDFEBER; GLUZ, 2011).

Desde este momento comenzaron a implementarse acciones orientadas por la inclusión de los sectores desplazados del sistema educativo para cumplir con la obligatoriedad escolar hasta la finalización del nivel secundario, como estableció la LEN. En cuanto a la EJA, desde el año 2004, se desarrollaron acciones destinadas a la alfabetización de jóvenes y adultos en todo el país y de terminalidad educativa. Dos años después, estos avances se incorporaron en la actual Ley de Educación Nacional (LEN) al dar tratamiento a la Educación Permanente de Jóvenes y Adultos (EPJA) como una de las diferentes modalidades que tiene el sistema educativo formal. Con ello, se desplazó la antigua consideración de la EJA como régimen especial y preparación instrumental para la inserción laboral hacia un nuevo reconocimiento orientado a la diversidad y articulación con el mundo del trabajo a partir su legitimación dentro del sistema formal (HISSE, 2009). En el marco de la extensión de la obligatoriedad de la educación secundaria, las acciones desplegadas en el nivel medio de la modalidad de Jóvenes y Adultos adquirieron centralidad y un importante impacto en el crecimiento de la matrícula. En 2008, la cantidad de estudiantes inscriptos fue de 430.595; en 2009, ascendió 475. 943 y en 2010, alcanzó los 522.289. Por su parte, el nivel secundario concentró en la última década el mayor porcentaje de estudiantes de EDJA, entre el 60 y 70\% (ARGENTINA, 2012).

Como parte de un curso más amplio de políticas educativas de revinculación, ingreso, permanencia y terminalidad escolar, en 2008 surgió el Plan de Finalización de estudios Primarios y
Secundarios (PLAN FINES) para jóvenes y adultos, cuyo objetivo fue garantizar el cumplimiento de la obligatoriedad escolar, en complementación con las acciones desplegadas desde el área de la Educación de Jóvenes y Adultos. El diseño del plan muestra que el Estado Nacional concibió el derecho a la educación en todos sus niveles y modalidades educativas conjuntamente con la proyección de prácticas y de estrategias vinculadas a la continuidad de los estudios superiores.

En los años 2000, momento de importante avance de las políticas sociales en el marco del gobierno del presidente Luiz Inácio Lula da Silva se produjo la creación del Fondo de Mantenimiento y Desarrollo de la Educación Básica y la valorización de los Profesionales de la Educación (FUNDEB), que amplió las restricciones impuestas por el FUNDEF, creado por Fernando Henrique Cardoso y pasó a considerar la Educación Infantil, la Enseñanza Fundamental y Media. También en esos años el Ministerio de Educación asumió la defensa del piso nacional salarial básico de los salarios de los profesores de la Educación Básica y la organización de la Conferencia Nacional de Educación (OLIVEIRA, 2009). Otra de las transformaciones significativas fue la anulación del Decreto ${ }^{\circ} 2.208$ de 1997, también del gobierno de Fernando Henrique Cardoso, que de acuerdo con la LDB reestableció la posibilidad de integrar curricularmente el nivel medio y la enseñanza técnico-profesional. Aunque esa iniciativa fue reconocida como un avance, algunos sectores alertaron sobre la necesidad sancionar una Ley de Educación Profesional y Tecnológica, como instancia superadora de la fragmentación del sistema educativo, más que impulsar la implementación de programas focalizados y contingentes, entre los cuales se menciona al PROEJA (FRIGOTTO; CIAVATTA; RAMOS, 2005).

En relación al sistema educativo, la Enmienda Constitucional no 59/2009 alteró el status previsto para el Plan Nacional de Educación (PNE) y lo transformó en una exigencia constitucional establecida por períodos decenales. El PNE pasó a ser un eje organizador del sistema educativo nacional al que deben articularse los planos elaborados por los niveles subnacionales del gobierno de la educación. En relación a la EJA, las novedades se produjeron principalmente en la implementación de acciones que impulsan la integración entre la formación profesional y la enseñanza fundamental o media, entre ellas la 


\section{en Argentina y el PROEJA en Brasil}

creación del PROEJA a mediados de los años 2000. El último Plan Nacional de Educación (Ley $\mathrm{n}^{\circ}$ 13.005/2014), previsto para el periodo 2014-2024 fortaleció de esa dirección. Así lo indicó la $10^{\circ}$ meta: "ofrecer un mínimo de $25 \%$ de las matrículas de la Educación de Jóvenes y Adultos, en la enseñanza fundamental y media, en forma integrada a la educación profesional" (BRASIL, 2014). Otra de las metas de ese Plan Nacional también contempló la alfabetización de jóvenes y adultos, proponiendo elevar la tasa de alfabetización de la población con 15 años y más al 93,5\% hasta el 2015, aún no alcanzada y con una ambiciosa propuesta de resolver el problema del analfabetismo absoluto y de reducir en un 50\% la tasa del analfabetismo funcional (BRASIL, 2014).

\section{Las nuevas propuestas educativas: el Plan FINES en Argentina y el PROEJA en Brasil}

\section{Un análisis del Plan FINES 2 como programa educativo}

El Plan FINES es un programa de "terminalidad" de estudios secundarios que permite la finalización, el inicio o el reingreso a la escuela en cualquier año del nivel. En sus comienzos convocó a jóvenes y adultos que terminaron de cursar la educación secundaria y adeudan materias con una modalidad de tutorías. Posteriormente, amplió el grupo de destinatarios hasta alcanzar a todas las personas mayores de 18 años que deseen terminar los estudios secundarios con una modalidad de cursada presencial por tramos (FINES 2). En este sentido, el programa intenta revertir el proceso de exclusión que reproduce la escuela secundaria y diseña una propuesta que promueve el "retorno a la escuela".

Desde su inicio, el programa tuvo una amplia convocatoria. En 2009, las cifras divulgadas por las autoridades educativas nacionales indicaron que había 200.000 inscriptos y que en un año se habían graduados más de 70.000 estudiantes. ${ }^{2}$ En 2014, el Ministro de Educación afirmó que habían egresado 500 mill jóvenes y adultos a través del FINES y que del total, el $90 \%$ de los egresados eran alumnos que debían algunas materias, mientras que sólo el $10 \%$ restante habían iniciado o completado sus estudios secundarios. ${ }^{3}$ Esta amplia cobertura del plan expresa las demandas por obtener acreditaciones escolares de los sectores populares de la población, las que no resultaban satisfechas a través de la escuela secundaria convencional.
El programa busca la universalización del derecho a la educación a través de una estrategia de acción afirmativa o discriminación positiva al dar tratamiento o incorporar en su propuesta educativa necesidades y posibilidades de algunos grupos poblacionales socioeconómicamente más vulnerables, específicamente jóvenes y adultos excluidos del sistema educativo tradicional. La orientación a grupos- meta fue la tendencia predominante de política social en la década de los '90, justificada por un discurso que sostenía la necesidad de concentrar los medios disponibles en los sectores más necesitados de la población. Asumieron un sentido compensatorio constituyendo en el espacio escolar subjetividades "tuteladas" (DUSCHATZKY; REDONDO, 2000). En contraste, el Plan FINES expresa una transición desde una concepción compensatoria-focalizada de política social imperante en las reformas neoliberales de la década de 1990 a otra con vocación universalista y centrada en la "contraprestación" al Estado (JACINTO, 2010; GLUZ; RODRÍGUEZ MOYANO, 2012)

El Programa surge en el marco del debate educativo por diseñar y generar nuevas modalidades alternativas que tiendan a incorporar a los jóvenes y adultos provenientes de los sectores más vulnerables de la población. Así, el régimen académico del Plan FINES2 asume cierta flexibilidad: carácter presencial con un $75 \%$ de asistencia obligatoria, asistencia a clase dos veces por semana, aprobación de cinco materias cuatrimestrales durante tres años, posibilidad de establecer turnos de mañana, tarde y vespertino según la demanda de los estudiantes.

Al tratarse de un plan nacional, en el marco de un sistema educativo organizado descentralizadamente, los contenidos curriculares, por ejemplo en la provincia de Buenos Aires, corresponden a los diseños para los Bachilleratos para Adultos.

Los docentes que participan deben entregar una propuesta pedagógica acorde con una selección y adecuación de estos contenidos, asimismo el plan admite la posibilidad de que cada alumno siga su propio recorrido, independientemente del bloque de materias que esté cursando en el cuatrimestre. La asistencia está flexibilizada y en la mayoría de los casos se tramita directamente en la relación docente-alumno, al acordar las estrategias pedagógicas que permiten compensar las ausencias justificadas de los estudiantes. En sintonía, en el sistema de promoción de la materia existe una 
importante asociación entre asistencia y aprobación (LÓPEZ, 2015). A estas características, estrechamente vinculadas con la posibilidad de completar los estudios en paralelo con las obligaciones laborales y familiares, se suman a otras como por ejemplo, que las madres o los padres pueden llevar a sus hijos a clase (CUESTAS, 2014).

Las clases se desarrollan en diferentes "sedes", organizadas de manera descentralizada, que se encuentran ancladas territorialmente en barrios periféricos, permitiendo un mayor acceso de los sectores excluidos de la escuela media tradicional. De esta manera, la flexibilidad en la propuesta se traduce en el plano institucional (JACINTO; BURGOS; ROBERTI; 2014), dado que las sedes pueden asumir múltiples espacios (entorno escolares o extraescolares) donde se reconocen diferencias en la infraestructura material. Esta apertura de entornos no convencionales posee la ventaja de aproximar a la educación a los propios territorios, pero tiene como contrapartida ciertas limitaciones, porque muchos espacios no fueron diseñados para la práctica educativa (LÓPEZ, 2015).

Los cambios en el formato escolar están orientados hacia la facilitación de la permanencia y de la conclusión de los estudios y acompañados por estrategias pedagógicas en sintonía con el perfil socio-cultural de los destinatarios. El formato produce ciertas habilitaciones centradas en la tríada acceder-permanecer-finalizar (LÓPEZ，2015). En este sentido, la modalidad que asume el plan FINES 2 a la vez marca sus límites en cuanto a la posibilidad de diseñar políticas que impliquen una transformación integral de la escuela secundaria tendiente a revertir su carácter tradicionalmente excluyente.

El Programa expresa, además, un rasgo "intersectorial" que asumen las políticas socioeducativas luego de la crisis socioeconómica y política que atravesó la Argentina en 2001, al constituirse en una iniciativa conjunta entre el Ministerio de Educación de la Nación, el Ministerio de Desarrollo Social, el Consejo Nacional de Educación, los Ministerios de Educación de las jurisdicciones provinciales, la Dirección Nacional de la EJA y los Centros Educativos de Nivel Secundario-CENS (Resolución Consejo Federal de Educación $\left.n^{\circ} 917 / 08\right)^{4}$. En ese sentido, busca articular distintos dispositivos desplegados desde las áreas gubernamentales de desarrollo social, trabajo y educación, a fin de promover el desarrollo inclusivo de la población, por lo que se inscribe en una dimensión más amplia de la política social ${ }^{5}$. Desde la perspectiva de la modalidad de la EJA, el Programa también rescata y continúa la tradición de "intersectorialidad" propia de su configuración institucional y de sus dinámicas de expansión, a diferencia de otras modalidades del sistema educativo (FINNEGAN; BRUNETTO, 2014).

En relación con el modelo de gestión, el FINES 2 logra visibilizar una modalidad relativamente reciente de organización y funcionamiento del Estado nacional en nuestro país. Por un lado, éste asume el papel principal de garantizar el derecho a la educación. Para ello, financia los gastos operativos en las escuelas-sede y el pago de los salarios docentes (estipulados en horas cátedra semanales), realiza capacitaciones para equipos técnicos provinciales, la provisión de materiales para profesores tutores y estudiantes y promueve en colaboración con los ministerios jurisdiccionales de la apertura de sedes y de seguimiento y evaluación del Plan. Por otra parte, el formato de gestión también combina la descentralización con la ampliación de la autonomía local. Los ministerios educativos jurisdiccionales adoptan distintas responsabilidades: fijan las escuelas sedes del Plan, organizan tutorías y capacitación de profesores tutores, secretarios docentes y directivos y rinden cuentas al Estado nacional sobre los pagos de salarios y gastos operativos. A nivel local, el gobierno municipal adquiere un papel clave en la implementación del programa, dado que define los requisitos y la afectación de los "referentes", quienes coordinan el trabajo en las sedes, previa solicitud a la Inspección distrital de la apertura del centro.

De esta manera, se trata de un tipo de estado "transversal" que rompe tanto con los clásicos compartimentos fijos y aislados que escinden sus planos nacional-subnacional y públicoprivado como con un sistema de gestión exclusiva, por lo que adopta un papel cualitativamente distinto en la articulación horizontal de esos componentes (OSZLAK, 2001). La noción refiere a una forma estatal que cruza las distintas instancias y/o niveles jurisdiccionales (nacional, territorial y local) y fija nuevas reglas de interacción entre ellos y en relación a la distribución del poder y la división de responsabilidades funcionales (OSZLAK, 2001).

Desde el enfoque de la acción pública, el Programa puede interpretarse como "un espacio socio-político construido [...] por la proliferación de sus objetivos, actores e instrumentos" (FONTAINE, 2015, p.140), lo que lo configura en tanto orden 


\section{en Argentina y el PROEJA en Brasil}

negociado, donde las influencias entre Estado y sociedad no son unilaterales. Así, es posible identificar un esquema de toma de decisiones que opera "más en la horizontalidad o en la circularidad que en la obediencia a una concepción lineal y jerárquica" (FELDFEBER; OLIVEIRA, 2012,p. 12).

Sumado a lo anterior, la multiplicidad de participantes involucrados en el Programa ocurre principalmente en dos niveles: en el de las agencias educativas y de desarrollo social (nacional, provincial y municipal) y entre los actores estatales y sociales, quienes a través de un convenio con el Ministerio de Educación de la Nación pueden ser sedes del Plan. Entre los participantes destacan instituciones propias de la modalidad, como las de la EJA, incluidas los Centros Educativos de Nivel Secundario (CENS), instituciones educativas de gestión privada y pública, sindicatos, organizaciones de la sociedad civil, universidades, iglesias de diversos credos y en menor medida, empresas. Como resultado de la menor jerarquización y no linealidad del Programa, sobresale cierta naturaleza fragmentada, compleja y flexible de la acción pública (FELDFEBER; OLIVEIRA, 2012, p. 12).

Por otra parte, la heterogeneidad de intervenciones, perspectivas y objetivos perseguidos por actores con diversos intereses permite comprender a nivel local/distrital las distintas reformulaciones en el diseño del Plan como en sus orientaciones y variantes en el curso de acción. Al respecto, algunos autores observan que la interdependencia compleja entre actores que movilizan racionalidades políticas y lógicas de acción vinculadas al sistema educativo formal, a la educación popular, a la dinámica de la política social y al campo de las organizaciones sociales y populares, entre otras, permite tensionar las tradiciones selectivas del nivel secundario y desafiar el predominio de los regímenes académicos y formatos escolares tradicionales al interior de la EJA (FINNEGAN; BRUNETTO, 2014).

El surgimiento y posterior desarrollo del Programa se inscribe en una nueva matriz de relaciones entre el Estado nacional y las organizaciones sociales y políticas. Muchas de éstas últimas surgen con el propósito de generar alternativas y resistencias a las políticas neoliberales implementadas durante la década de 1990 en el país. Entre 2003 y 2015, en el marco de los gobiernos peronistas y de corte progresista de Néstor y Cristina Kirchner, numerosas organizaciones comienzan a participar en programas sociales y educativos. Algunas investigaciones recientes, señalan que las organizaciones involucradas en el Plan FINES 2 configuran su relación con el Estado tanto en términos de aportantes de "territorialidad" como resultado de su militancia y participación en las redes políticas locales- como también en clave de "brazo ortopédico", vinculado a su papel accesorio del (y en el) Estado (DI BASTIANO, 2014). En ese sentido, los actores sociales y políticos participantes en el Plan FINES 2 robustecen la acción estatal en el territorio, a la vez que participan de un espacio de recursos que promueve distintas formas políticas.

\section{EI PROEJA en Brasil}

El PROEJA es un programa nacional que busca integrar la escolaridad de los niveles educativos a la formación profesional, entendiendo que la formación no es exclusiva del mundo del trabajo o del mundo de la educación y concibiéndola como una intersección de diferentes contribuciones y abordajes, incluyendo las de los sujetos trabajadores (BRASIL, 2007a, p. 46). La articulación entre esas dos instancias constituye la referencia principal de este programa.

Inicialmente, el Decreto $\mathrm{n}^{\circ}$ 5.478/2005 creó un programa de integración restringido al nivel medio. Posteriormente, el Decreto $\mathrm{n}^{\circ} 5.840$, del 13 de julio de 2006 extendió su alcance y lo transformó en el Programa Nacional de Integración de la Educación Profesional con la Educación Básica en la modalidad EJA (PROEJA), incluyendo tanto el nivel de enseñanza fundamental como media. Las pautas y orientaciones nacionales para ese programa están contenidas principalmente en dos documentos denominados Documentos Base, focalizado en cada nivel educativo (BRASIL, 2007a; 2007b).

Este programa también busca contribuir a la universalización del derecho a la escolarización extendiendo los servicios educativos en esa modalidad y disponibilizando espacios para jóvenes y adultos históricamente excluidos de los servicios educativos nacionales. Tanto el FINES como el PROEJA parten de la necesidad reconocida de oportunizar el acceso y la permanencia a los servicios educativos de jóvenes y adultos de los sectores más postergados de la sociedad. El diagnóstico producido por los Documentos Base del PROEJA señala la necesidad de generar una oferta educativa que supere las fragilidades y déficits históricos de la EJA así como la importancia de 
innovar a través de una integración entre formación profesional y enseñanza escolar correspondiente a los niveles educativos (BRASIL, 2007a).

El PROEJA pretende, por lo menos en el espíritu de la letra que lo orienta, reposicionar a la EJA y constituirla simultáneamente en una modalidad educativa y en una estrategia de formación continua (BRASIL, 2007a). En este sentido, se entiende que la oferta del PROEJA se distancia de la función remedial y compensatoria, originaria de la EJA, para asumir las funciones de reparación en relación al acceso a servicios educativos y de superación de las desigualdades educativas, como ya señalaban las Directrices Curriculares para la EJA del año 2000.

En relación al PROEJA de nivel medio, la apuesta va aún un poco más a fondo, pues se postula el abandono de una perspectiva reducida de formación para el mercado de trabajo para asumir una formación integral que rompa con la visión exclusivamente propedéutica de un nivel medio marcado fuertemente por la pérdida de su especificidad para convertirse en una instancia preparatoria para el examen de ingreso a la universidad (vestibular) en las escuelas regulares que prestan servicios educativos en ese nivel de enseñanza (BRASIL, 2007a).

En relación al nivel de enseñanza fundamental, el PROEJA busca asociar el aumento de la escolaridad a una formación profesional básica, por lo menos inicialmente, con la pretensión de que esta instancia tienda a desaparecer por la universalización del acceso y la permanencia de los estudiantes en las escuelas denominadas regulares (BRASIL, 2007b).

Como uno de los documentos de ese programa enfatiza, se busca a través del PROEJA recuperar el papel regulador del Estado nacional, responsable por el diseño y la promoción de políticas públicas que impulsen una mejora de la educación pública, en consonancia con estados y municipios; en relación al financiamiento, se señala la importancia de los recursos presupuestarios provenientes del FUNDEB (BRASIL, 2007a; 2007b).

La normativa apunta que dicho programa puede ser ofrecido por las instituciones de la Red Federal de Educación Profesional, Científica y Tecnológica, escuelas de la Red Estadual o Municipal, o por otras instituciones privadas nacionales vinculadas al sistema sindical (SISTEMA S). Algunos análisis enfatizan que la participación de las instituciones de la Red Federal de Educación Profesional, Científica y Tecnológica se enfatiza que si bien la normativa señala una invitación a participar del PROEJA, en el plano fáctico esa implementación es compulsoria, pues para esos espacios se establecieron porcentajes de vacantes que deben ser garantizados (FRIGOTTO; CIAVATTA; RAMOS, 2005; OLIVEIRA; FERREIRA, 2010; FRANZOI et al, 2010; MOURA; ENRIQUE, 2012). El trabajo de Ferreira y Oliveira (2010) enfatiza que no se trata de cuestionar la relevancia de ese programa, que promueve la inserción de la EJA en espacios institucionales públicos que habitualmente adoptaron prácticas selectivas y sí de analizar las condiciones para que el programa puede cumplir con la finalidad de promoción de la inclusión social.

A diferencia del FINES de Argentina, que, como ya fue señalado, se construye como un espacio intersectorial, el PROEJA busca producir una articulación entre espacios institucionales del propio sistema educativo que el programa propone que funcionen en forma articulada: "El gran desafío de esa política es la construcción de una identidad propia para nuevos espacios educativos, inclusive de una escuela de/para jóvenes y adultos" (BRASIL, 2007a, p. 46). Como señalan Moura y Enrique (2012), la integración de tres espacios históricamente distantes (la educación fundamental, la formación profesional técnica y la EJA) configura uno de sus principales desafíos. En ese sentido, el PROEJA constituye una tentativa de interrupción y reconfiguración de tradiciones institucionales que históricamente operaron de forma disociada. Moura y Enrique (2012) también enfatizan que un desafío mayor de esa propuesta es constituir a la EJA a partir de otras tareas que no son exclusivamente las de la formación escolar inicial: "podrá centrarse en la formación continuada de la clase trabajadora y, por tanto, en la capacitación para el ejercicio de actividades más complejas dentro de las profesiones técnicas de nivel medio e, inclusive, como forma de contribuir para el acceso a la educación superior" (MOURA; ENRIQUE, 2012).

Los documentos base del PROEJA establecen orientaciones que pautan los fundamentos político-pedagógicos, los modos de organización de la oferta incluyendo referencias a una organización de tiempos y espacios diferenciada de aquellos habitualmente establecidos por las escuelas convencionales. Se subraya la necesidad de organización de tiempos adecuados a las distintas realidades, así como la consideración de los ritmos y saberes previos, desde que se 


\section{en Argentina y el PROEJA en Brasil}

alcancen los objetivos previstos en el currículum para cada etapa o ciclo (BRASIL 2007a).

También los documentos referidos al nivel fundamental y medio, contemplando las especificidades de cada uno, fijan cargas horarias, así como principios de la construcción curricular, orientada por la propuesta de integración entre calificación profesional y aumento de la escolaridad $\mathrm{y}$ las formas en que tiene que ser entendida la evaluación. Algunos análisis critican dichas orientaciones en relación a ciertas incoherencias en relación a las definiciones de cargas horarias que pautan un punto máximo y no el mínimo de horas establecidas. Además, se apunta que, siendo una propuesta innovadora, se enfrenta a problemas semejantes al de la EJA más tradicional, como la interrupción de la escolaridad y que algunas instituciones buscan alternativas para enfrentarlos, como la reevaluación de metodologías o el apoyo de sectores especializados (FRANZOI et al, 2010). En definitiva, los formatos escolares, aunque flexibilizados, parecen continuar operando.

\section{Conclusiones}

Este estudio, al que caracterizamos como inicial y exploratorio, permitió identificar que tanto el FINES como el PROEJA surgen como iniciativas orientadas inicialmente a la escolaridad de nivel medio y, posteriormente, amplían su alcance a la denominada educación básica, comprendiendo ambos niveles educativos. Las dos propuestas emergen varios años después del reconocimiento legal de la EJA como modalidad educativa; en la LDB de 1996 y con las Directrices Curriculares para la EJA del 2000, en Brasil; y en la Ley de Educación Nacional de 2006 en Argentina. Puede decirse que los tiempos de las políticas de EJA en general son demorados.

Ambos programas emergen a partir de contextos marcados por políticas públicas que buscan potencializar el acceso y la permanencia de amplios sectores excluidos de la población a los sistemas educativos y de demandas por la creación de diseños alternativos que contemplen las realidades con las que la EJA trabaja. En este sentido, proponen condiciones generales más realistas y una flexibilización de algunas normas reguladoras de las escuelas convencionales. Además, responden a un movimiento que parte de la identificación de déficits históricos en los servicios educativos de la EJA y los dos casos pretenden producir ampliaciones y adecuaciones de la oferta educativa.

Sin embargo, los caminos trazados por las políticas educativas de ambos países presentan importantes diferencias. En Brasil el programa se articula a una importante redefinición de los espacios institucionales del propio sistema educativo buscando integrar la enseñanza escolar de los niveles con la formación profesional. Esto implica producir aproximaciones e integraciones entre tradiciones institucionales diferentes, a partir de trayectorias institucionales construidas en forma disociada. En este sentido la propuesta es tan interesante como arriesgada, por las tensiones producidas entre los principios y orientaciones pretendidos por el PROEJA y ciertas "inercias" propias de las instituciones escolares. En Argentina, el Plan FINES 2 amplía claramente el alcance territorial de la EJA y su penetración en los distintos territorios a través de alianzas estratégicas con los diferentes sectores sociales y una perspectiva intersectorial de las políticas. Sin embargo, la apuesta se limita a la terminalidad educativa, sin considerar aspectos de la formación asociada al mundo del trabajo, como en el caso brasileño.

Ambas propuestas, con las limitaciones y desafíos que enfrentan, no dejan de ser novedosas para una Educación de Jóvenes y Adultos que evidencia, en su versión tradicional, un claro conjunto de limitaciones.

\section{Notas}

1 Aclaramos que todas las traducciones del portugués al español, presentadas en este texto, son de nuestra autoría.

2 Como los datos sobre la cobertura del Plan FINES no están disponibles, se recurrió a publicaciones periodísticas de un periódico argentino que publicó declaraciones del entonces Ministro de Educación, Alberto Sileoni. Diponibles on line en: http://www.lanacion.com.ar/1154658-sileoni-hayque-terminar-con-la-rigidez-del-secundario

3 Materia periodística disponible on line en: http://www.telam.com.ar/notas/201404/59797alberto-sileoni-plan-fines-educacion.html

4 Se crea en 2008 a través de la Resolución 917/08 del Ministerio de Educación de la Nación y la Resolución 66/08 del Consejo Federal de Educación, concertado en la Mesa Federal de Educación Permanente de Jóvenes y Adultos y se encuentra a cargo de la Dirección Nacional de Educación de Jóvenes y Adultos.

5 Tanto el Programa Asignación Universal por Hijo 
(AUH) como el Programa de Respaldo a Estudiantes de Argentina (Progresar) son dos ejemplos de tales articulaciones. El primero consiste en asignación destinado a trabajadores desocupados y empleados informales. Se percibe un monto de 966 pesos mensuales (alrededor de 68 US\$) por cada hijo menor de 18 años (hasta un tope de cinco, hijos) y de por vida para aquellos que sufran alguna discapacidad. Como contraprestación de la asignación uno de los requisitos es la asistencia escolar. El segundo consiste en acciones integradas que permitan capacitar a los jóvenes entre 18 y 24 años a fin de completar la escolaridad obligatoria, iniciar o facilitar la continuidad de una educación superior y realizar experiencias de formación y/o prácticas calificantes en ambientes de trabajo.

\section{Referencias}

ARGENTINA. Ley n ${ }^{\circ} 1420$ de Educación Común, del 8 de julio de 1884. Ley de Educación Común.

Ley $\mathrm{n}^{\circ} 24.195$, de 29 de abril de 1993. Ley Federal de Educación.

Ley $n^{\circ} 24.049$, del 2 de enero de 1992. Faculta al Poder Ejecutivo Nacional para transferir servicios educativos a las provincias.

Ley $\mathrm{n}^{\circ} 24.521$, de 7 de agosto de 1995. Ley

Nacional de Educación Superior.

. Ministerio de Educación. Dirección Nacional de Evaluación de la Calidad Educativa (DINIECE). Relevamiento Anual 2003.

. Ley $\mathrm{n}^{\circ} 26.206,14$ de diciembre de 2006. Ley de Educación Nacional.

. Ministerio de Educación. Dirección Nacional de Evaluación de la Calidad Educativa (DINIECE). Relevamiento Anual 2012.

Ley $n^{\circ} 27.045$, del 3 de diciembre de 2014. Modifica la Ley $n^{\circ} 26.206$ estableciendo la obligatoriedad escolar a partir de los cuatro años.

BEISEGEL, C. Considerações sobre a política da união para a educação de jovens e adultos analfabetos. Revista Brasileira de Educação, n. 4, jan-abr 1997, p. 26-34.

BRASIL. Constituição Federal de 1988.

Promulgada em 5 de outubro de 1988. Disponível em http://www.planalto.gov.br/ccivil_03/constituica o/constituição.htm. Aceso em 20-03-2016

BRASIL. Lei no 5,379, de 15 de dezembro de 1967. Institui a Fundação Movimento Brasileiro de Alfabetização (MOBRAL).

Lei $\mathrm{n}^{\circ}$ 5.692, de 11 de agosto de 1971. Diretrizes e Bases da Educação Nacional.

Lei $\mathrm{n}^{\circ}$ 9.394, de 20 de dezembro de 1996. Lei de Diretrizes e Bases da Educação Nacional.

Ministério de Educação. Parecer CNE/CEB n $^{\circ} 11 / 2000$. Diretrizes para a Educação de Jovens e Adultos.

. Decreto $\mathrm{n}^{\circ} 5.478$, de 24 de junho de 2005. Institui, no âmbito das instituições federais de educação tecnológica, o Programa de Integração da Educação Profissional ao Ensino Médio na Modalidade de Educação de Jovens e Adultos PROEJA. Brasília, DF: 24 de junho de 2005.

. Decreto ${ }^{\circ}$ 5.840, de 13 de julho de 2006. Institui, no âmbito federal, o Programa Nacional de Integração da Educação Profissional com a Educação Básica na Modalidade de Educação de Jovens e Adultos - PROEJA, e dá outras providências. Brasília, DF: 13 de julho de 2006.

Ministério da Educação. Documento base Programa de Integração da Educação Profissional Técnica de Nível Médio ao Ensino Médio na Modalidade de Educação de Jovens e Adultos PROEJA. Brasília: SETEC, 2007a.

. Ministério da Educação. Documento base Programa de Integração da Educação Profissional Técnica do Ensino Fundamental ao Ensino Médio na Modalidade de Educação de Jovens e Adultos PROEJA. Brasília: SETEC, 2007b.

Emenda Constitucional $n^{\circ} 59$, de 11 de novembro de 2009. Acrescenta $3^{\circ}$ ao art. 76 do Ato das Disposições Constitucionais Transitórias e outras disposições.

Lei $n^{\circ} 13.005$, de 25 de junho de 2014. 
Aprova o Plano Nacional de Educação. en Argentina y el PROEJA en Brasil

Anuario Brasileiro da Educação Básica 2016. Todos pela Educação. Disponible em: http://www.todospelaeducacao.org.br/. Acceso en 29-07-2016

BRASLAVSKY, C., La discriminación educativa en la Argentina, Buenos Aires: FLACSO-GEL, 1985.

CUESTAS, P. Yo quiero mi título. La perspectiva de los alumnos y alumnas del Plan FINES II de La Plata sobre su paso por esta experiencia educativa. Anales. Argentina en el escenario latinoamericano actual: debates desde las ciencias sociales, Universidad Nacional de La Plata, Facultad de Humanidades y Ciencias de la Educación, La Plata, 2014. http://jornadassociologia.fahce.unlp.edu.ar/vii i-jornadas-2014/PONmesa38Cuestas.pdf/view? Acesso en 25-01-2016

DE LA FARE, M. Principales ideas, discusiones y producciones en educación de Jóvenes y adultos en Argentina: aportes para una reconstrucción histórica. Serie Informes de Investigación, 2. Buenos Aires: DINIECE/Ministerio de Educación de la Nación, 2010. Disponible en: http://www.me.gov.ar/diniece/. Acesso en 5-012016

DE LA FARE, M; PEREIRA, M. V. Educación de Jóvenes y Adultos: políticas educativas nacionales y experiencias de Educación Popular en Argentina y Brasil en los años 60 e inicios de los 70. Pátzcuaro/México: Revista Interamericana de Educación de Adultos, CREFAL, n. 1, enero-junio, 2011, p. 6-24.

DE LA FARE, M. Luz Vieira Mendez, la UNESCO y el lanzamiento del Programa Nacional Intensivo de Alfabetización y Educación de Adultos (PNIAyEA) en Argentina (1965). In: SUASNÁBAR, C.; CAMPOS, N. (Orgs.) Educação no Brasil e na Argentina: escritos de história intelectual. Ponta Grossa/Paraná: Editora Universidade Estadual de Ponta Grossa, 2013, p. 295-311.

DI BASTIANO, R. El brazo ortopédico del Estado. Reflexiones en torno a una experiencia de participación de una organización social en la implementación del Plan FINES 2 en la ciudad de
La Plata. Anales. Argentina en el escenario latinoamericano actual: debates desde las ciencias sociales, Universidad Nacional de La Plata, Facultad de Humanidades y Ciencias de la Educación, La Plata, 2014. Disponible en: http://jornadassociologia.fahce.unlp.edu.ar/viiijornadas-2014/PONmesa15DiBastiano.pdf/view? Acceso en 14-04-2016.

DI PIERRO, Clara. Um balanço da evolução recente da educação de jovens e adultos no Brasil. In: Construção coletiva: contribuições à educação de jovens e adultos. - Brasília: UNESCO, MEC, RAAAB, 2005, p. 17-30.

DUSCHATZKY, S.; REDONDO, P. Las marcas del Plan Social Educativo o los indicios de ruptura de las políticas públicas. In: DUSCHATZKY, S (comp.) Tutelados y Asistidos; Programas sociales, políticas públicas y subjetividad. Buenos Aires: Paidós, 2000, p. 121-185.

FELDFEBER, M.; OLIVEIRA, D. A. Nuevas y viejas formas de regulación de los sistemas educativos en Brasil y Argentina. Las políticas para el nivel secundario en argentina: ¿hacia una educación más igualitaria? Montevideo: Revista Uruguaya de Ciencia Política, n. 1, v. 1, 2012, p. 25-43

FELDFEBER, M.; GLUZ, N. Las políticas educativas en Argentina: herencias de los '90, contradicciones y tendencias de "nuevo signo", Campinas, Educação \& Sociedade, vol. 32, núm. 115, abril-junio, 2011, pp. 339-356

Fernández, M., Lemos, M. y Wiñar, L. La Argentina Fragmentada. El caso de la Educación. Buenos Aires: Miño y Dávila, 1997.

FERREIRA, E.B; OLIVEIRA, E. C. Entre a inclusão social e a integração curricular: os dilemas políticos e epistemológicos do PROEJA. Educação $\&$ Realidade, $\mathrm{n}$.

35(10, jan/abr 2010, p. 87-108.

FERREIRA, E. B.; FONSECA, M. O planejamento das políticas educativas no Brasil e seus desafios atuais. Perspectiva, Florianópolis, v. 29, n. 1, p. $69-$ 96, jan./jun. 2011.

FERREIRA, E. B.; OLIVEIRA, E. C. Entre a inclusão social e a integração curricular: os dilemas 
políticos e epistemológicos do PROEJA. Educação \& Realidade, n. 35 (1), p. 87-108, jan/abr., 2010.

FINNEGAN, F.; BRUNETTO, C. "Acá hay un trabajo político": Actores y relaciones en la producción del Plan FINES 2 Secundaria en la Provincia de Buenos Aires. Anales. VIII Jornadas de Sociología de la UNLP, Ensenada: 3 al 5 de diciembre de 2014. Disponible en: http://www.memoria.fahce.unlp.edu.ar/trab_eventos /ev.4364/ev.4364.pdf. Acceso en 13-02-2016

FONTAINE, G., El análisis de políticas públicas. Conceptos, Teorías y Métodos, Barcelona: Antrhopos; Quito: FLACSO, 2015.

FRANZOI, N.; HYPOLITO, A.; FISCHER, A.; PINO, M.; SANTOS, S.V. Escola, saberes e trabalho: a pesquisa do PROEJA no Rio Grande do Sul. Educação \& Realidade, Porto Alegre, n. 35 (1), p. 167-186, jan/abr. 2010.

FRIGOTTO, G.; CIAVATTA, M.; RAMOS, M. A política de Educação Profissional no governo Lula: um percurso histórico controvertido. Educação \& Sociedade, Campinas, v. 26, n. 92, p. 1087-1113, Especial - out. 2005.

\section{GLUZ, N. Y RODRÍGUEZ MOYANO, I.}

Asignación Universal por Hijo, condiciones de vida y educación. Las política sociales y la inclusión escolar en la provincia de Buenos Aires. Archivos Analíticos de Políticas Educativas, 21 (21),p. 1-28, 2013.Disponible en: http://epaa.asu.edu/ojs/article/ view /1102. Acceso en12-04-2016

HADDAD, S. A educação de pessoas jovens e adultas e a nova LDB. In: BRZEZINSKI, I. (Org.) LDB interpretada: diversos olhares se entrecruzam. São Paulo, Cortez, 1999, p. 29-40.

HADDAD, S.; DI PIERRO, C. Escolarização de Jovens e adultos. Revista Brasileira de Educação, $\mathrm{n}^{\circ}$ 14, maio/jun/jul/ago, 2000, p. 108-130.

HISSE, M.C.(Coord.). Educación Permanente de Adultos y Jóvenes. Serie Recomendaciones para la elaboración de diseños curriculares. Buenos Aires: Ministerio Nacional de Educación,2009. Disponible en: http://repositorio.educacion.gov.ar/dspace/bitstr eam/handle/123456789/89816/Jovenes\%20y\%20ad ultos.pdf?sequence=1. Acesso en 12-04-2016.
JACINTO, C. Introducción. Elementos para un marco analítico de los dispositivos de inserción laboral de jóvenes y su incidencia en las trayectorias. In: JACINTO, C. La construcción de las trayectorias laborales de jóvenes; políticas, instituciones, dispositivos y subjetividades. Buenos Aires: Teseo, 2010, p. 15-50.

JACINTO, C.; BURGOS, A. Y ROBERTI, E. (2014). Formación en y para el trabajo de grupos focalizados de población. Anales Seminario: Los programas de educación, formación y empleo de jóvenes en el marco de los nuevos paradigmas de protección social. Departamento de Investigaciones Educativas, Cinvestav, Sede Sur. México, D.F., 28 y 29 de agosto de 2014.

LÓPEZ, E. El formato escolar en políticas de inclusión educativa: el caso del FINES 2. Anales $V$ congreso Nacional e Internacional de estudios comparados en Educación. Buenos Aires, 24 al 26 de junio de 2015.

MOURA, D. H.; HENRIQUE, A.L.S. PROEJA: entre desafios e possibilidades. Natal: Holos, ano 28 , v. 2, 2012, p. 114-129.

OLIVEIRA, D. A. As políticas educacionais no governo Lula: rupturas e permanências. RBPAE, v.25, n.2, p. 197-209, mai./ago. 2009.

OLIVEIRA, R. P. Da universalização do Ensino fundamental ao desafio da qualidade: uma análise histórica. Campinas: Educação \& Sociedade, v. 28, n. 100 - Especial, out. 2007, p. 661-690.

OSZLAK, O. Hacia un estado transversal: el caso argentino. Buenos Aires: Encrucijadas Revista de la Universidad de Buenos Aires, año uno, n. 6., abril 2001, p. 1-14

SUASNÁBAR, C. ; ROVELLI, L. Gouvernance et réformes de l'éducation en Argentine récemment. Revue internationale d'éducation de Sèvres. Sèvres: Centre international d'études pédagogiques, n. 55, 2010, p. 17-26.

TIRAMONTI, G.; SUASNABAR, C. (2000). La reforma educativa nacional. En busca de una interpretación. Aportes, Buenos Aires, n. 15, p. 1931, 2000. 


\section{Sobre as autoras}

Mónica de la Fare: Doutora em Serviço Social e Pós-doutora em Educação (PUCRS). Atua desde 2013 como professora do Programa de Pós-graduação em Educação da Escola de Humanidades da PUCRS.

Laura Rovelli: Doutora em Ciências Sociais. Atua como pesquisadora do IdIHCS-CONICET e professora em nível de graduação e pós-graduação na Faculdade de Humanidades e Ciências da Educação da Universidade Nacional de La Plata.

Daniela Atairo: Doutora em Ciências Sociais. Atua como pesquisadora do IdIHCS e professora em nível de graduação e pós-graduação, na Faculdade de Humanidades e Ciências da Educação da Universidade Nacional de La Plata.

Recebido em agosto de 2016.

Aprovado em novembro de 2016. 